\title{
Implementing visual cues to improve the efficacy of screening colonoscopy: exploiting the Hawthorne effect
}

\section{Eric Omar Thena, Christopher Branaa, Sriharsha Dadanaa, Srikanth Maddikaa, Andrew Ofosu ${ }^{b}$, Sabrina Brana ${ }^{a}$, Tina Wexlera, Tagore Sunkarab $^{b}$, Andrea Culliford ${ }^{a}$, Vinaya Gaduputia}

SBH Health System, New York; The Brooklyn Hospital Center, Clinical Affiliate of The Mount Sinai Hospital, Brooklyn, NY, USA

\section{Abstract}

Background Colonoscopy is the gold standard for colon cancer screening. Adenoma detection rate and a withdrawal time of $6 \mathrm{~min}$ are quality metrics to measure the efficacy of colonoscopy in colon cancer screening. The aim of our study was to exploit the Hawthorne effect in an effort to ensure adherence to a minimum 6-min withdrawal time and subsequently increase adenoma detection rate.

Methods This was a retrospective single-center study where we reviewed the records of patients who underwent screening colonoscopy in 2015 and 2017. We divided our patient population into 2 groups. The first group of patients from 2015 underwent screening colonoscopy with no visual cues on the colonoscopy monitor. The second group of patients from 2017 had visual cues indicating withdrawal time on the colonoscopy monitor.

Results Screening colonoscopy had a statistically significantly higher adenoma detection rate when performed with visual cues compared to without visual cues ( $25.3 \%$ vs. $19.45, \mathrm{P}=0.04)$. Polyp detection rate was also higher in the group where visual cueing was used $(52.9 \%$ vs. $22.9 \%, \mathrm{P}<0.001)$. There were no statistically significant differences in actual withdrawal time or cecal intubation rates.

Conclusion Visual cues indicating withdrawal time are a useful intervention that results in an increased adenoma detection rate. Given its practicality and cost effectiveness, we recommend universally implementing visual cues to ensure adherence to a minimum 6-min withdrawal time.

Keywords Adenoma detection rate, polyp detection rate, cecal intubation rate, colorectal cancer, colonoscopy

Ann Gastroenterol 2020; 33 (4): 1-5

\section{Introduction}

Colon cancer is the third leading cause of cancer deaths worldwide [1]. In an effort to prevent these deaths, current

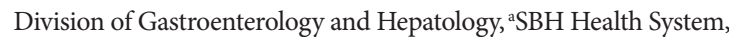
New York (Eric Omar Then, Christopher Brana, Sriharsha Dadana, Srikanth Maddika, Sabrina Brana, Tina Wexler, Andrea Culliford, Vinaya

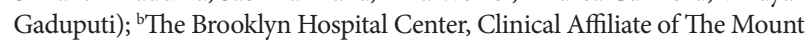
Sinai Hospital, Brooklyn, NY (Andrew Ofosu, Tagore Sunkara), USA

Conflict of Interest: None

Correspondence to: Eric Omar Then, MD, 4422 Third Avenue, Bronx, NY 10457 USA, e-mail: ericomarthen27@yahoo.com

Disclosures: The work described in this manuscript was presented at the American College of Gastroenterology's Scientific Meeting in Philadelphia, Pennsylvania, 2018

Received 15 January 2020; accepted 27 February 2020; published online 10 May 2020

DOI: https://doi.org/10.20524/aog.2020.0491 guidelines suggest every person undergo colon cancer screening starting at age 50; the gold standard for this is colonoscopy [2]. In order to quantify the efficiency of colonoscopy as a screening tool, one must look at the adenoma detection rate (ADR). $\mathrm{ADR}$ is a metric representing the proportion of colonoscopies performed that result in the detection of one adenoma [3]. With a high ADR, adenomas are identified and resected earlier, which in turn results in a lower incidence of colon cancer. Because of this, many strategies are being implemented to increase $\mathrm{ADR}$ and subsequently decrease cause-specific mortality from colon cancer.

A colonoscopy is divided into 2 phases. The first is the insertion phase, where the endoscopist navigates through peristaltic waves to reach the illeocecal junction. The second phase is the withdrawal phase, where colonic lesions are more easily identified and treated. ADR will vary from physician to physician, but one quality measure that is uniform in increasing ADR is a minimum 6-min withdrawal time [4]. Our study aimed to expand on this quality measure. By incorporating visual cues onto the monitor where the colonoscopy is displayed, we intended to exploit Hawthorne and novelty 
effects to ensure each endoscopist indeed achieved a minimum 6-min withdrawal time, and subsequently improve ADR.

\section{Patients and methods}

This was a retrospective single-center observational study. The study took place over the course of 2 years, specifically 2015 and 2017. The study was performed according to the guidelines indicated by the declaration of Helsinki and was approved by the Institutional Review Board (IRB) of St. Barnabas Hospital Health System.

\section{Patient selection}

Data were collected from the electronic medical records of patients and tabulated in Microsoft Excel. The study included patients who underwent colonoscopy and were seen in our outpatient gastroenterology clinic. These patients were seen in 2015 and 2017, and underwent screening colonoscopy for colorectal cancer (CRC) by one of 4 gastroenterologists on our staff. Patients were excluded from our study if they had a poorly prepped colonoscopy, as documented by one of our gastroenterologists. Patients were also excluded if they underwent flex sigmoidoscopy rather than full colonoscopy. Similarly, patients who had missing information, namely documented withdrawal time, age, family history of colon cancer or smoking status, were also excluded from our study.

\section{Evaluation of results}

Our study sought out to establish a correlation between a Hawthorne effect and an increased ADR. We aimed to establish this correlation by adding a visual cue to our endoscopy screens indicating withdrawal time (Figs. 1 and 2). We then compared the ADR between colonoscopies performed with the visual cue and those performed without.

\section{Endoscopic equipment}

All colonoscopies at our institution were performed using high definition Olympus PCF- Q180AL and PCF-PH190L colonoscopes (Olympus America, Center Valley, PA).

\section{Group division}

The control group consisted of patients who underwent colonoscopy in 2015 without the visual cue on the endoscopy screen indicating withdrawal time. The study group consisted of patients who underwent colonoscopy in 2017, when visual cues indicating withdrawal times were implemented at our institution.

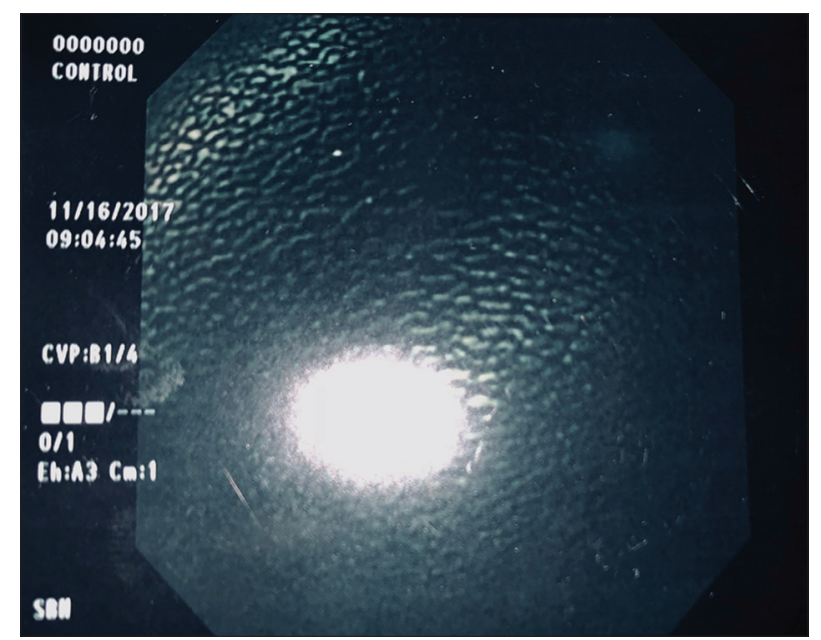

Figure 1 Control group colonoscopy camera display including (from left side, top to bottom): medical record number, date, time (for display purposes only)

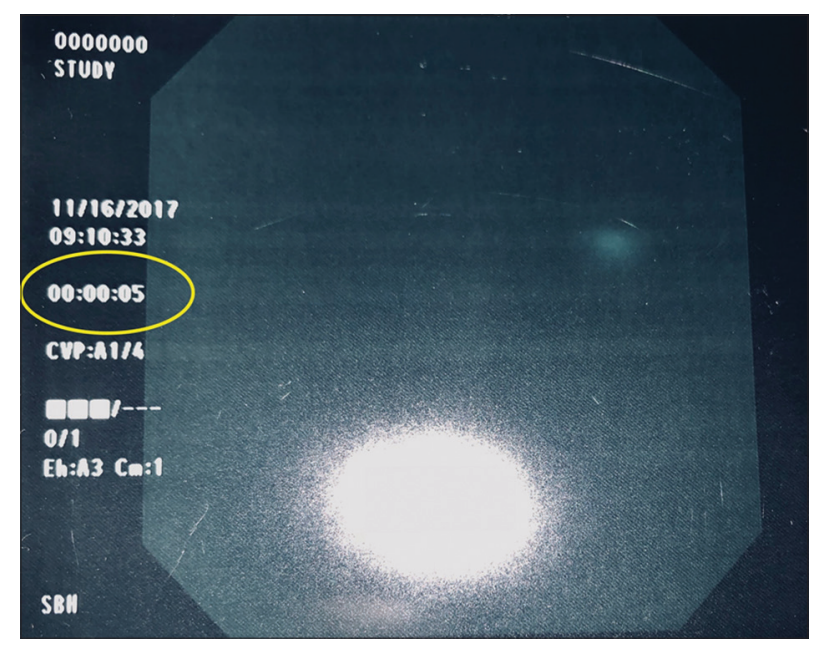

Figure 2 Study group colonoscopy camera display including (from left side, top to bottom): medical record number, date, time, withdrawal time (circled) (for display purposes only)

\section{Data collection}

Baseline demographic data, including age, sex and ethnicity, were collected for all patients in the study. We collected data on the patients' body mass index (BMI), alcohol ingestion, tobacco usage and co-morbid conditions such as diabetes mellitus, hypertension, and hyperlipidemia. Additionally we analyzed cecal intubation rates and withdrawal time in minutes between both study and control groups. Finally, we calculated $\mathrm{ADR}$ and polyp detection rates (PDR) for both groups.

\section{Statistical analysis}

Results were reported as means with standard deviations for most variables. For comparisons of continuous variables between the 2 study groups we used the $t$-test. Categorical variables for the 
2 study groups were compared by chi-square analysis. A 2-tailed P-value of $<0.05$ was considered to be statistically significant.

\section{Results}

In total there were 851 patients who met our inclusion criteria. Of these patients, 406 were included in our control group, and 445 were included in our study group. Baseline demographic characteristics for both cohorts are listed in Table 1 . The mean age was 59.5 years for our control group and 58.7 years for our study group. There was no statistically significant difference in age between the 2 groups $(\mathrm{P}=0.1)$. Similarly, there was no statistically significant difference in BMI (29.53 vs. 29.57, $\mathrm{P}=0.9$ ). In our control group $58.6 \%$ of patients were female, and $41.4 \%$ were male. Similar findings were seen in our study group, where $56.2 \%$ of patients were female and $43.8 \%$ were male. The majority of patients in both groups were Hispanic (61.6\% in control group, 59.1\% in study group), followed by African American (37.4\% in control group, 39.1\% in study group) and Caucasian ( $0.8 \%$ in control group, $0.5 \%$ in study group).

Comorbid conditions for our patients are listed in Table 2. We found that there was no statistically significant difference between our control group and study group in terms of patients with hypertension ( $53.4 \%$ vs. $54.2 \%, \mathrm{P}=0.8$ ), diabetes mellitus ( $28.5 \%$ vs. $30.3 \%, \mathrm{P}=0.5$ ), tobacco use ( $87.9 \%$ vs. $83.8 \%, \mathrm{P}=0.1$ ), or a family history of colon cancer $(6.9 \%$ vs. $5.8 \%, \mathrm{P}=0.5)$.

Quality indicators for both cohorts are listed in Table 3. We found that there was a statistically significantly greater ADR in our study group when compared to our control group (25.3\% vs. $19.4 \%, \mathrm{P}=0.04$ ). Additionally, we found that there was a statistically significantly greater PDR in our study group when compared with our control group $(52.9 \%$ vs. $22.9 \%, \mathrm{P}<0.01)$. We found that there was no statistically significant difference between our control group and study group with regard to withdrawal time (7.19 vs. $7.21 \mathrm{~min}$, $\mathrm{P}=0.8)$ or cecal intubation rates $(96.8 \%$ vs. $98.2 \%, \mathrm{P}=0.1)$.

\section{Discussion}

In our study we found that using a visual cue in the form of a withdrawal timer added to our displays resulted in a statistically significantly increased ADR in 2017, compared to the ADR with no withdrawal timer in 2015. Additionally, we found a statistically significantly increased PDR in the group with the withdrawal timer, compared to the group without the withdrawal timer. We did not find any statistically significant differences in actual withdrawal time or cecal intubation rates. We attribute these findings to a Hawthorne effect taking place in our study group.

The Western Electrical Company's Hawthorne Works first discovered the Hawthorne effect, sometimes referred to as a researcher effect or observer effect, in the 1920s. Australian born psychologist Elton Mayo, who sought to improve productivity at the electrical plant, first conceived the study. Through a series of experiments, Mayo and his team found that productivity increased in groups who believed they were being observed and individually evaluated for performance. This was initially used in
Table 1 Baseline demographic characteristics

\begin{tabular}{lccc}
\hline Variable & $\begin{array}{c}\text { Control group } \\
(\mathrm{N}=406)\end{array}$ & $\begin{array}{c}\text { Study group } \\
(\mathrm{N}=444)\end{array}$ & P-value \\
\hline Age (+/- SD) & $59.52(8.39)$ & $58.76(7.75)$ & 0.1659 \\
BMI (+/- SD) & $29.53(5.61)$ & $29.57(5.87)$ & 0.9188 \\
Sex & & & \\
$\quad$ Male & $168(41.4 \%)$ & $195(43.8 \%)$ & 0.4877 \\
Race & & & \\
African American & $152(37.4 \%)$ & $174(39.2 \%)$ & 0.6214 \\
Hispanic & $250(61.6 \%)$ & $263(59.2 \%)$ & 0.5276 \\
Caucasian & $3(0.8 \%)$ & $2(0.5 \%)$ & 0.6740 \\
Other & $1(0.2 \%)$ & $5(1.1 \%)$ & 0.2199 \\
\hline
\end{tabular}

BMI, body mass index

Table 2 Comorbid conditions

\begin{tabular}{lccc}
\hline Variable & $\begin{array}{c}\text { Control group } \\
(\mathrm{N}=406)\end{array}$ & $\begin{array}{c}\text { Study group } \\
(\mathrm{N}=444)\end{array}$ & P-value \\
\hline HTN & $217(53.45 \%)$ & $241(54.28 \%)$ & 0.8364 \\
DM & $116(28.57 \%)$ & $135(30.34 \%)$ & 0.5984 \\
HLD & $126(31.11 \%)$ & $132(29.66 \%)$ & 0.7091 \\
FH of colon cancer & $28(6.9 \%)$ & $26(5.8 \%)$ & 0.5749 \\
Tobacco use & $357(87.93 \%)$ & $373(83.82 \%)$ & 0.1146 \\
\hline
\end{tabular}

HTN, hypertension; DM, diabetes mellitus; HLD, hyperlipidemia; FH, family history

Table 3 Quality indicators

\begin{tabular}{lccc}
\hline Variable & $\begin{array}{c}\text { Control group } \\
(\mathrm{N}=406)\end{array}$ & $\begin{array}{c}\text { Study group } \\
(\mathrm{N}=444)\end{array}$ & P-value \\
\hline ADR & $79(19.45 \%)$ & $113(25.39 \%)$ & 0.0402 \\
PDR & $93(22.9 \%)$ & $235(52.92 \%)$ & 0.0001 \\
WT (+/- SD) & $7.19(1.36)$ & $7.21(1.66)$ & 0.8549 \\
CI & $393(96.80 \%)$ & $437(98.20 \%)$ & 0.1728 \\
\hline
\end{tabular}

$A D R$, adenoma detection rate; $P D R$, polyp detection rate; $W T$, withdrawal time; CI, cecal intubation

the industrial domain to improve productivity, but quickly gained value in clinical research with the objective of improving clinical practice and care [5]. As seen in our study, using a simple time cue with nurse monitoring, physicians performing colonoscopies found more polyps and adenomas than when they performed colonoscopies where cueing was not used. By exploiting the Hawthorne effect, we found that visual cueing during colonoscopy increases the effectiveness of screening/surveillance colonoscopy and may potentially contribute to decreasing rates of CRC and improving the overall quality of patient care.

In 2015 the American Society for Gastrointestinal Endoscopy (ASGE) and the American College of Gastroenterology (ACG) defined quality indicators for colonoscopy. These quality indicators are divided into pre-procedure, intra-procedure, and post-procedure phases. Considering the nature of our study, this discussion will center on the intra-procedure phase. Quality indicators for the 
intra-procedure phase include: intubation of the entire colon with detailed mucosal inspection, cecal intubation, and-more relevant to our study-a withdrawal time of 6 min [6]. To further expand on quality indicators of the intra-procedure phase, one important measure to analyze is ADR. As mentioned previously, ADR is defined as the proportion of colonoscopies performed that result in the detection of one or more adenomas. As per the ASGE and ACG, every gastroenterologist should aim to have at least a $20 \% \mathrm{ADR}$ in females and $30 \%$ in males during routine screening colonoscopy [6]. In addition to decreasing the risk of CRC on initial screening colonoscopy, an increased ADR has also been linked to a decreased risk of interval CRC, defined as a CRC diagnosed within 60 months after a negative colonoscopy [7]. One study by Corley et al found that $\mathrm{ADR}$ was inversely associated with the risks of interval CRC, advanced stage interval CRC, and fatal interval CRC [8]. Withdrawal time also plays an integral role in ADR and decreasing the risk of CRC. In 2006, Barclay et al conducted a 15-month retrospective study that involved 7882 colonoscopies in a large community based, outpatient practice. They found that higher ADRs were seen in endoscopists who had longer mean withdrawal times [4]. With our study findings we hope to implement universal visual cues as part of the quality indicators to decrease the incidence of CRC and interval CRC alike.

A study published by Nielsen et al mirrored our findings. In their study they performed a total of 307 colonoscopies and recorded PDR and withdrawal time under 3 different clinical scenarios. In the first scenario, the staff performing colonoscopies were unaware of the objective of the study. In the second scenario the staff were encouraged to aim for a minimum withdrawal time of $8 \mathrm{~min}$. Finally, in the third scenario, they assessed retention of knowledge one year after the second scenario. They found that PDR almost doubled from the first to the second scenario, and that knowledge gained from the intervention was retained one year later in the third scenario [9]. This further validates our findings and demonstrates the importance of non-conventional means to increase adherence to quality measures.

Other seldom-discussed metrics that merit consideration are PDR and cecal intubation rate (CIR). In our study we did not find a statistically significant difference between the control and study groups in CIR, but we did observe a statistically significantly greater PDR in our study group when compared to our control group. In clinical practice PDR is less regarded than ADR, but is still noteworthy. This, in part, is due to a proportion of polyps being diagnosed as hyperplastic polyps, which have no malignant potential compared to adenomas. Indeed studies have shown that over 95\% of CRCs arise from benign adenomas [10]. Despite this, PDR may play a role in predicting ADR. One meta-analysis that comprised 25 studies found that a ratio of 0.68 could be used to calculate ADR from PDR [11]. Another study by Francis et al had similar findings. By examining the results from 3367 colonoscopies, they found that a ratio of 0.64 predicted ADR from PDR [12]. Perhaps equally as important as predicting ADR is the detection of proximal serrated polyps. Although the majority of CRCs arise from benign adenomas via the adenoma-carcinoma sequence, a significant proportion may arise from serrated polyps. With the discovery of the serrated neoplastic pathway, through which serrated polyps progress to CRC, more emphasis has been placed on recognizing these lesions [13]. Serrated polyps of the proximal colon in particular have been shown to be more prevalent in interval CRC [14]. The importance of proximal PDR is underscored by a study conducted by Boroff et al, who found that PDR was more accurate in predicting ADR in the right colon compared to the sigmoid colon and rectum [15].

A variable that may have contributed to the findings in our study is withdrawal technique rather than actual withdrawal time. Lee et al conducted a study that sought to establish a relationship between these 2 variables. In order to evaluate withdrawal technique they adapted a score from Rex et al. This scoring system accounted for fold examination, lumen distension and cleansing [16]. When comparing high-ADR endoscopists with low-ADR endoscopists they found a statistically significant difference in technique scores, but no difference in withdrawal times [17]. In our study, considering there was no statistically significant difference in withdrawal times between both groups, it is possible that the increased ADR was attributed to withdrawal technique.

Our study had a number of limitations. The first was its retrospective nature, which bears inherent weaknesses. Secondly, our data was extracted from a single center that had a predominantly minority population (Hispanics and African Americans), and hence could potentially not be applicable to predominantly Caucasian patient populations. Strengths of our study included completion of documentation regarding withdrawal time, cecal intubation, and evaluation of polyp histology by an expert pathologist. Further studies should be pursued to validate our findings.

In conclusion we found that adding a visual timer cue on the display monitors of colonoscopies resulted in increased PDR and more importantly, ADR. We propose the universal implementation of visual cueing on all monitors to ensure adherence to a minimum 6-min withdrawal time and encourage adequate withdrawal technique. By doing so, we hope to increase ADR and consequently decrease deaths from CRC.

\section{Summary Box}

\section{What is already known:}

- Colorectal cancer is the third leading cause of cancer deaths worldwide

- Increased adenoma detection rate and a withdrawal time of a minimum 6 min are quality indicators for screening colonoscopy that have been associated with a decreased risk of colorectal cancer

- Measures are being implemented to attempt to increase adenoma detection rate and consequently decrease the risk of development of colorectal cancer

\section{What the new findings are:}

- Implementing a visual cue indicating withdrawal time was associated with a statically significant increased adenoma detection rate and polyp detection rate

- Universal implementation of a visual cue may reduce the risk of development of colorectal cancer 


\section{References}

1. Marley AR, Nan H. Epidemiology of colorectal cancer. Int J Mol Epidemiol Genet 2016;7:105-114.

2. Bibbins-Domingo K, Grossman DC, Curry SJ, et al; US Preventive Services Task Force. Screening for Colorectal Cancer: US Preventive Services Task Force Recommendation Statement. JAMA 2016;315:2564-2575.

3. Kaminski MF, Regula J, Kraszewska E, et al. Quality indicators for colonoscopy and the risk of interval cancer. $N$ Engl J Med 2010;362:1795-1803.

4. Barclay RL, Vicari JJ, Doughty AS, Johanson JF, Greenlaw RL. Colonoscopic withdrawal times and adenoma detection during screening colonoscopy. N Engl J Med 2006;355:2533-2541.

5. McCarney R, Warner J, Iliffe S, van Haselen R, Griffin M, Fisher P. The Hawthorne Effect: a randomised, controlled trial. BMC Med Res Methodol 2007;7:30.

6. Rex DK, Schoenfeld PS, Cohen J, et al. Quality indicators for colonoscopy. Am J Gastroenterol 2015;110:72-90.

7. Lee YM, Huh KC. Clinical and biological features of interval colorectal cancer. Clin Endosc 2017;50:254-260.

8. Corley DA, Jensen CD, Marks AR, et al. Adenoma detection rate and risk of colorectal cancer and death. $N$ Engl J Med 2014;370:1298-1306.

9. Nielsen $A B$, Nielsen $\mathrm{OH}$, Hendel J. Impact of feedback and monitoring on colonoscopy withdrawal times and polyp detection rates. BMJ Open Gastroenterol 2017;4:e000142.

10. Bond JH. Polyp guideline: diagnosis, treatment, and surveillance for patients with colorectal polyps. Practice Parameters Committee of the American College of Gastroenterology. Am J Gastroenterol 2000;95:3053-3063.

11. Niv Y. Polyp detection rate may predict adenoma detection rate: a meta-analysis. Eur J Gastroenterol Hepatol 2018;30:247-251.

12. Francis DL, Rodriguez-Correa DT, Buchner A, Harewood GC, Wallace M. Application of a conversion factor to estimate the adenoma detection rate from the polyp detection rate. Gastrointest Endosc 2011;73:493-497.

13. Leggett B, Whitehall V. Role of the serrated pathway in colorectal cancer pathogenesis. Gastroenterology 2010;138:2088-2100.

14. Kahi CJ, Hewett DG, Norton DL, Eckert GJ, Rex DK. Prevalence and variable detection of proximal colon serrated polyps during screening colonoscopy. Clin Gastroenterol Hepatol 2011;9:42-46.

15. Boroff ES, Gurudu SR, Hentz JG, Leighton JA, Ramirez FC. Polyp and adenoma detection rates in the proximal and distal colon. Am J Gastroenterol 2013;108:993-999.

16. Rex DK. Colonoscopic withdrawal technique is associated with adenoma miss rates. Gastrointest Endosc 2000;51:33-36.

17. Lee RH, Tang RS, Muthusamy VR, et al. Quality of colonoscopy withdrawal technique and variability in adenoma detection rates (with videos). Gastrointest Endosc 2011;74:128-134. 